\title{
Combating COVID-19 With Mesenchymal Stem/Stromal Cell Therapy: Promise and Challenges
}

\author{
Shihua H. Wang ${ }^{1}$, Ashok K. Shetty ${ }^{2}$, Kunlin Jin ${ }^{3}$ and Robert Chunhua Zhao ${ }^{1,4 *}$ \\ ${ }^{1}$ Institute of Basic Medical Sciences Chinese Academy of Medical Sciences, School of Basic Medicine Peking Union Medical \\ College, Beijing, China, ${ }^{2}$ Institute for Regenerative Medicine and Department of Molecular and Cellular Medicine, Texas A\&M \\ University College of Medicine, College Station, TX, United States, ${ }^{3}$ Department of Pharmacology and Neuroscience, \\ University of North Texas Health Science Center Fort Worth, Fort Worth, TX, United States, ${ }^{4}$ Department of Cell Biology, \\ School of Life Sciences, Shanghai University, Shanghai, China
}

Keywords: mesenchymal stem cell, COVID-19, therapy, extracellular vesicle, exosome

OPEN ACCESS

Edited by:

Erdal Karaoz,

Istinye University, Turkey

Reviewed by:

Philippe Bourin,

Independent researcher, Toulouse,

France

Simone Pacini

University of Pisa, Italy

*Correspondence:

Robert Chunhua Zhao

zhaochunhua@ibms.pumc.edu.cn

Specialty section:

This article was submitted to

Stem Cell Research,

a section of the journal

Frontiers in Cell and Developmental

Biology

Received: 09 November 2020 Accepted: 09 December 2020

Published: 05 January 2021

Citation:

Wang SH, Shetty AK, Jin K and Chunhua Zhao R (2021) Combating COVID-19 With Mesenchymal Stem/Stromal Cell Therapy: Promise and Challenges.

Front. Cell Dev. Biol. 8:627414.

doi: 10.3389/fcell.2020.627414
There is no effective therapy for COVID-19 currently. Until an efficient vaccine is developed, therapeutic strategies that facilitate faster recovery in COVID-19 patients developing life-threatening complications are urgently needed. From this perspective, mesenchymal stem/stromal cells (MSCs) that have been used in the clinic for moderating the immune system in graft vs. host disease (GVHD) (Fisher et al., 2019), type 2 diabetes (Path et al., 2019), autoimmune diseases (Weiss and Dahlke, 2019), spinal cord injury (Shende and Subedi, 2017), and several other diseases deserve consideration for treating COVID-19. Importantly, MSCs lack angiotensin-converting enzyme-2 (ACE2) receptor, which is a receptor widely distributed on the surface of human cells, and required for the entry of coronavirus into host cells. Such property ensures that injected MSCs can accomplish immunomodulatory effects without being destroyed by the virus. Our clinical trial, which is also the first published report, showed that the intravenous injection of human umbilical cord-derived MSCs eased the cytokine storm syndrome (CSS) and significantly improved the outcome in severe COVID-19 patients (Leng et al., 2020), suggesting the promise of MSC therapy for saving lives of COVID-19 patients developing severe complications. Although we repeatedly emphasize that studies in a larger cohort of patients are urgently needed to validate this promising therapeutic intervention, some businesses are taking advantage of our findings and offer cell therapy for COVID-19 patients, using types of cells that might not have been tested vigorously for safety and efficacy in FDA-approved clinical trials. This unethical commercial use of MSCs is criticized by Leigh Turner in his article entitled "Preying on Public Fears and Anxieties in a Pandemic: Businesses Selling Unproven and Unlicensed Stem Cell Treatments for COVID-19" published in Cell Stem Cell (Turner, 2020), which we read with great interest and are inspired to write this opinion paper. We strongly agree that such businesses could pose significant risks to patients and detract efforts to advance evidence-based stem cell therapy for COVID-19. We must emphasize that there are no approved MSC-based approaches for the prevention or treatment of COVID-19 patients, although several FDA-approved clinical trials are ongoing at present. Furthermore, we would like to suggest some guidelines from regulatory agencies that might stop such businesses from selling stem cell therapy to COVID-19 patients. Such guidelines should: (1) require companies to provide detailed scientific information on the safety and efficacy of mesenchymal stem/stromal cells in preclinical trials; clear criteria of MSCs manufacture and quality control; scientific rationale of organizing a clinical trial using stem cell therapy for patients; the qualifications of the principal investigators and all the medical staff; registered information 


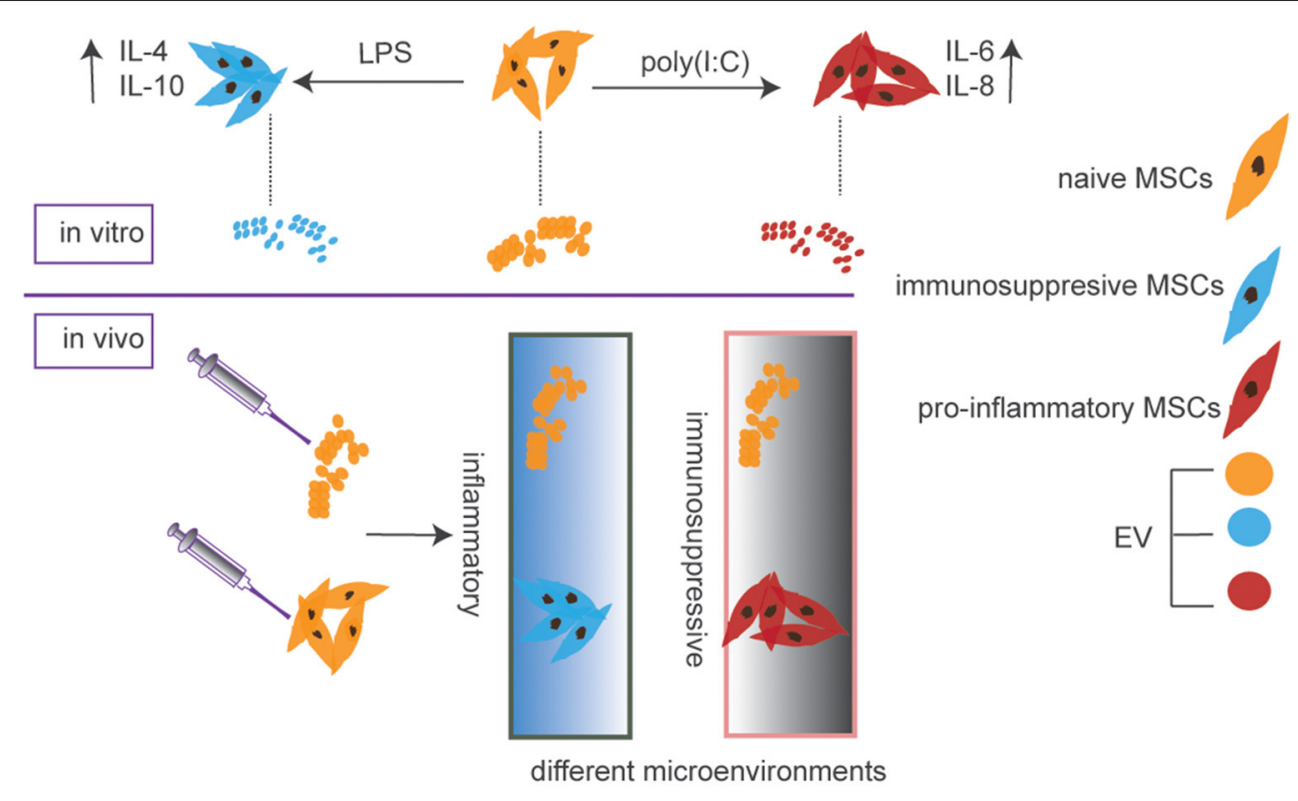

FIGURE 1 | EV cargos are determined by the status of parental MSCs and would not change after the injection, while MSCs could alter secretome after homing to sites of injury or inflammation due to their ability for plasticity depending on the microenvironment they come across.

in clinical trial https://clinicaltrials.gov/. (2) promote central or local government to establish an independent stem cell therapy committee that could update latest research outcomes or provide professional suggestions for patients. (3) encourage the public to report unproven stem cell therapy to the state administration so that the illegal company could be punished. The objective of these guidelines is to help patients and their caretakers to enroll only in randomized clinical trials conducted in renowned hospitals with approvals from the local Institutional Review Board (IRB) and the Federal Drug Administration (FDA). We think generating and implementing a new policy or law at the national level could stop such cell treatment to COVID-19 patients. Besides, the social platform, including media, should publicize and educate the public regarding dangers associated with cell treatment offered by businesses that are not FDA-compliant.

In addition to MSCs, some businesses are also selling MSCderived extracellular vehicles (EVs) for COVID-19 therapy. EVs, nanosized vesicles secreted by virtually all cells for intercellular communication, carry a cargo comprising cytokines, growth factors, lipids, and microRNAs (Robbins and Morelli, 2014; Phinney and Pittenger, 2017; Kalluri and LeBleu, 2020). Studies in disease models have suggested that stem cell-derived EVs exert similar effects as their parental cells (Yanez-Mo et al., 2015; Kim et al., 2016). Because the therapeutic effects of MSCs have been attributed mainly to their secretome with a significant portion of which is disseminated through EVs (Rani et al., 2015; Keshtkar et al., 2018). MSC-derived EVs could be exploited for cell-free therapy. Compared to MSCs, EVs released from them have unique advantages such as more accessible storage and higher biosafety. However, the disadvantages include that
MSCs could home to sites of injury or inflammation and change their secretome depending on the local microenvironment. Importantly, MSCs are relatively large cells with an estimated average size of around $30 \mu \mathrm{m}$ in suspension (ranging from 16 to $53 \mu \mathrm{m}$ ) (Furlani et al., 2009; Leibacher and Henschler, 2016). This large size makes them easily trapped in lungs after intravenous administration, which might be a hurdle for the treatment of other diseases but a benefit for COVID-19 as lung is the major target organ of coronavirus. So, this specific and preferential pulmonary localization after administration is an advantage of MSCs compared to EVs in terms of treating COVID-19 or other lung diseases. On the other hand, the targeted delivery of EVs to intended tissues after an intravenous administration is currently challenging to achieve. More importantly, the cargo of EVs is determined by the status of parental MSCs as well as culture conditions (Figure 1), which do not change after interactions with the local microenvironment.

A very recent article reported the safety and efficacy of allogeneic bone marrow MSC-derived EVs in severe COVID19 patients who were already on hydroxychloroquine and azithromycin treatment. The study was a prospective, nonrandomized, open-label, investigation in which 24 SARS-CoV2 PCR positive patients at a single hospital center received MSC-derived EVs (Sengupta et al., 2020). Although such EV treatment did result in $71 \%$ of patients recovering from COVID19 , it was unclear whether the recovery could be attributed to EVs as the study lacked a matching control group and the protein or the microRNA composition of EVs employed was not reported in the study. One should be extremely cautious with clinical trials using EVs as their cargo determines the 
functional effects. Therefore, before MSC-derived EVs can be used in a clinical setting, standardized protocols for scaledup production, isolation, functional evaluation, and batchto-batch consistency need to be developed. These require a rigorous characterization of the composition of EVs generated in different batches using proteomics and small-RNA sequencing, the release criteria, and the biological properties. Also, the efficacy of EVs needs to be validated in animal models. While the MSC-derived EVs have the potential to replace MSC therapy for many conditions (Kim et al., 2016, 2020; Long et al., 2017), EV therapy does not seem ready in a short time.

In summary, we would like to emphasize that well-controlled, rationally designed clinical trials based on reliable scientific data are needed for both MSCs and MSCs-derived EVs for combating COVID-19.

\section{REFERENCES}

Fisher, S. A., Cutler, A., Doree, C., Brunskill, S. J., Stanworth, S. J., Navarrete, C., et al. (2019). Mesenchymal stromal cells as treatment or prophylaxis for acute or chronic graft-versus-host disease in haematopoietic stem cell transplant (HSCT) recipients with a haematological condition. Cochrane Database Syst. Rev. 1:CD009768. doi: 10.1002/14651858.CD009768.pub2

Furlani, D., Ugurlucan, M., Ong, L., Bieback, K., Pittermann, E., Westien, I., et al. (2009). Is the intravascular administration of mesenchymal stem cells safe? Mesenchymal stem cells and intravital microscopy. Microvasc Res. 77, 370-376. doi: 10.1016/j.mvr.2009.02.001

Kalluri, R., and LeBleu, V. S. (2020). The biology, function, and biomedical applications of exosomes. Science 367:eaau6977. doi: 10.1126/science.aau6977

Keshtkar, S., Azarpira, N., and Ghahremani, M. H. (2018). Mesenchymal stem cellderived extracellular vesicles: novel frontiers in regenerative medicine. Stem Cell Res. Ther. 9:63. doi: 10.1186/s13287-018-0791-7

Kim, D. K., Nishida, H., An, S. Y., Shetty, A. K., Bartosh, T. J., and Prockop, D. J. (2016). Chromatographically isolated CD63+CD81+ extracellular vesicles from mesenchymal stromal cells rescue cognitive impairments after TBI. Proc. Natl. Accad. Sic. U.S.A. 113, 170-175. doi: 10.1073/pnas.1522297113

Kim, H., Lee, M. J., Bae, E. H., Ryu, J. S., Kaur, G., Kim, H. J., et al. (2020). Comprehensive molecular profiles of functionally effective MSCderived extracellular vesicles in immunomodulation. Mol Ther. 28, 1628-1644. doi: 10.1016/j.ymthe.2020.04.020

Leibacher, J., and Henschler, R. (2016). Biodistribution, migration and homing of systemically applied mesenchymal stem/stromal cells. Stem Cell Res. Ther. 7:7. doi: 10.1186/s13287-015-0271-2

Leng, Z., Zhu, R., Hou, W., Feng, Y., Yang, Y., Han, Q., et al. (2020). Transplantation of ACE2- Mesenchymal stem cells improves the outcome of patients with COVID-19 pneumonia. Aging Dis. 11, 216-228. doi: $10.14336 / A D .2020 .0228$

Long, Q., Upadhya, D., Hattiangady, B., Kim, D. K., An, S. Y., Shuai, B., et al. (2017). Intranasal MSC-derived A1-exosomes ease inflammation, and prevent abnormal neurogenesis and memory dysfunction after status epilepticus. Proc. Natl. Accad. Sci. U.S.A. 114, E3536-E3545. doi: 10.1073/pnas.1703920114

Path, G., Perakakis, N., Mantzoros, C. S., and Seufert, J. (2019). Stem cells in the treatment of diabetes mellitus - focus on mesenchymal stem cells. Metabolism 90, 1-15. doi: 10.1016/j.metabol.2018.10.005

\section{AUTHOR CONTRIBUTIONS}

SW, AS, and RZ conceived, researched, and wrote the manuscript with input from KJ. All authors contributed to the article and approved the submitted version.

\section{FUNDING}

This work was funded by National Natural Science Foundation of China (Grant No. 81902848), the Fundamental Research Funds for the Central University (3332019062), CAMS Innovation Fund for Medical Sciences (2017-I2M-3-007), The National Key Research and Development Program of China (2016YFA0101000 and 2016YFA0101003), Beijing Key Laboratory of New Drug Development, and Clinical Trial of Stem Cell Therapy (BZ0381), National Institutes of Health (R01 NS106907 to AS).

Phinney, D. G., and Pittenger, M. F. (2017). Concise review: MSC-derived exosomes for cell-free therapy. Stem Cells 35, 851-858. doi: 10.1002/st em. 2575

Rani, S., Ryan, A. E., Griffin, M. D., and Ritter, T. (2015). Mesenchymal stem cellderived extracellular vesicles: toward cell-free therapeutic applications. Mol. Ther. 23, 812-823. doi: 10.1038/mt.2015.44

Robbins, P. D., and Morelli, A. E. (2014). Regulation of immune responses by extracellular vesicles. Nat. Rev. Immunol. 14, 195-208. doi: 10.1038/nr i3622

Sengupta, V., Sengupta, S., Lazo, A. Jr., Woods, P., Nolan, A., and Bremer, N. (2020). Exosomes derived from bone marrow mesenchymal stem cells as treatment for severe COVID-19. Stem Cells Dev. 29, 747-754. doi: $10.1089 / \mathrm{scd} .2020 .0080$

Shende, P., and Subedi, M. (2017). Pathophysiology, mechanisms and applications of mesenchymal stem cells for the treatment of spinal cord injury. Biomed. Pharmacother. 91, 693-706. doi: 10.1016/j.biopha.2017.04.126

Turner, L. (2020). Preying on public fears and anxieties in a pandemic: businesses selling unproven and unlicensed "stem cell treatments" for COVID-19. Cell Stem Cell 26, 806-810. doi: 10.1016/j.stem.2020.05.003

Weiss, A. R. R., and Dahlke, M. H. (2019). Immunomodulation by mesenchymal stem cells (MSCs): mechanisms of action of living, apoptotic, and dead MSCs. Front. Immunol. 10:1191. doi: 10.3389/fimmu.2019.01191

Yanez-Mo, M., Siljander, P. R., Andreu, Z., Zavec, A. B., Borras, F. E., Buzas, E. I., et al. (2015). Biological properties of extracellular vesicles and their physiological functions. J Extracell. Vesicl. 4:27066. doi: 10.3402/jev.v4. 27066

Conflict of Interest: The authors declare that the research was conducted in the absence of any commercial or financial relationships that could be construed as a potential conflict of interest.

Copyright (c) 2021 Wang, Shetty, Jin and Chunhua Zhao. This is an open-access article distributed under the terms of the Creative Commons Attribution License (CC $B Y)$. The use, distribution or reproduction in other forums is permitted, provided the original author(s) and the copyright owner(s) are credited and that the original publication in this journal is cited, in accordance with accepted academic practice. No use, distribution or reproduction is permitted which does not comply with these terms. 